\title{
The Standard Model particle content with complete gauge symmetries from the minimal ideals of two Clifford algebras
}

\author{
Niels G. Gresnigt ${ }^{\mathrm{a}}(\mathbb{D}$ \\ Department of Physics, Xi' an Jiaotong-Liverpool University, 111 Ren'ai Rd., Dushu Lake Science and Education Innovation District, Suzhou \\ Industrial Park, Suzhou 215123, People's Republic of China
}

Received: 6 April 2020 / Accepted: 10 June 2020 / Published online: 29 June 2020

(C) The Author(s) 2020

\begin{abstract}
Building upon previous works, it is shown that two minimal left ideals of the complex Clifford algebra $\mathbb{C} \ell(6)$ and two minimal right ideals of $\mathbb{C} \ell(4)$ transform as one generation of leptons and quarks under the gauge symmetry $S U(3)_{C} \times U(1)_{E M}$ and $S U(2)_{L}$ respectively. The $S U(2)_{L}$ weak symmetries are naturally chiral. Combining the $\mathbb{C} \ell(6)$ and $\mathbb{C} \ell(4)$ ideals, all the gauge symmetries of the Standard Model, together with its lepton and quark content for a single generation are represented. The combined ideals can be written as minimal left ideals of $\mathbb{C} \ell(6) \otimes \mathbb{C} \ell(4) \cong \mathbb{C} \ell(10)$ in a way that preserves individually the $\mathbb{C} \ell(6)$ structure and $\mathbb{C} \ell(4)$ structure of physical states. This resulting model captures many of the attractive features of the Georgi and Glashow $S U$ (5) Grand Unified Theory without introducing proton decay or other unobserved processes. Such processes are naturally excluded because they do not preserve the underlying algebraic structure.
\end{abstract}

\section{Introduction}

The Standard Model (SM) is currently the best model of particle physics. It provides a hugely predictive mathematical description of the most basic constituents of matter observed in nature, and their interactions via three of the four fundamental forces of nature. However, despite being able to use the theory to make remarkably accurate predictions, the theoretical origin of its mathematical structure remains unexplained. Specifically, why is the internal symmetry group of the SM what it is, when infinitely many other possibilities exist, and why do only some of the representations of the gauge groups correspond to physical states? Furthermore, why does the electroweak force depend on the chirality of physical states? Among others, these remain important open questions.

\footnotetext{
a e-mail: niels.gresnigt@xjtlu.edu.cn (corresponding author)
}

Grand unified theories (GUTs) merge the gauge groups of the SM into a single semi-simple Lie group. However, such GUTs, including the famous Georgi and Glashow's $S U(5)$ model and Georgi's Spin(10) model [1], invariably predict additional gauge bosons, interactions, and proton decay, none of which have thus far been observed. The background structure for these GUTs is $\mathbb{C} \ell(10)$, with the internal degrees of freedom of quarks and leptons described in terms of the irreducible representations of this algebra.

In [2], the basis states of two minimal left ideals of the Clifford algebra $\mathbb{C} \ell(6)$ were shown to transform precisely as a single generation of leptons and quarks under the electrocolor group $S U(3)_{C} \otimes U(1)_{E M}$. The Clifford algebra $\mathbb{C} \ell(6)$ there is generated from the left adjoint actions of the complex octonions $\mathbb{C} \otimes \mathbb{O}$ on itself. ${ }^{1}$ A Witt decomposition of $\mathbb{C} \ell(6)$ splits the algebra into a basis of nilpotent ladder operators, and the unitary symmetries that preserve this Witt decomposition are $S U(3)$ and $U(1)$. In other words, the minimal ideals are closed under the action of these unitary symmetry, and one finds that each basis state of the ideal transforms like a specific lepton or quark. In this scheme therefore, the basis states of the minimal left ideals are identified with physical states, and the gauge symmetries are those unitary symmetries that preserve the ideals.

Similarly, considering the left and right adjoint actions of the complex quaternions $\mathbb{C} \otimes \mathbb{H}$ each generates a distinct copy of $\mathbb{C} \ell(2)$, and allows one to describe the spin and chirality of leptons and quarks respectively. Combined, the left and right actions give a representation of the Dirac algebra

\footnotetext{
1 The application of octonions to quark symmetries goes back to the 1970s [3]. More recently division algebras have received some renewed interest. The results of [2] were extended to three generations recently by going beyond the octonions, and considering the next Cayley-dickson algebra of sedenions [4]. Dixon [5] showed that the composition algebra $\mathbb{R} \otimes \mathbb{C} \otimes \mathbb{H} \otimes \mathbb{O}$ plays a key role in the architecture of the SM. A gravitational theory based on a $\mathbb{R} \otimes \mathbb{C} \otimes \mathbb{H} \otimes \mathbb{O}$ metric has been constructed in [6].
} 
$\mathbb{C} \ell(4)[2,7]$. The minimal right ideals obtained from a Witt decomposition of $\mathbb{C} \ell(4)$ were previously also shown to transform as one generation of chiral fermions under $S U(2)_{L}$. The group $S U(2)$ that preserves the ideals in this case is found to automatically act on states of a single chirality [11].

In [7] it is shown that the individual $\mathbb{C} \ell(6)$ and $\mathbb{C} \ell(4)$ results can be combined, and that it is possible to represent one generation of chiral fermions (of fixed spin) with the SM gauge symmetries in terms of a single 32-dimensional $\mathbb{C} \ell(10)$ minimal ideal. However, this particular representation does not preserve the individual $\mathbb{C} \ell(6)$ and $\mathbb{C} \ell(4)$ ideal structures associated with specific leptons and quarks. This is a direct consequence of how the $S U(2)_{L}$ generators are defined, which does not account for the fact that the two particles in a weak doublet are elements of different $\mathbb{C} \ell(6)$ minimal left ideals. The $\mathbb{C} \ell(6)$ minimal left ideals are invisible to these $S U(2)_{L}$ generators meaning that the two particles making up a weak doublet must have the same $\mathbb{C} \ell(6)$ structure in this scheme, and differ only in their $\mathbb{C} \ell(4)$ structure.

This paper shows that by suitably redefining the $S U(2)_{L}$ generators in such a way that they simultaneously induce transformations within the $\mathbb{C} \ell(4)$ minimal right ideals but between different $\mathbb{C} \ell(6)$ minimal left ideals, it is possible to to embed all the physical states into minimal left ideals of $\mathbb{C} \ell(10)$ in a particularly aesthetic way that preserves both the individual $\mathbb{C} \ell(6)$ minimal left ideal and $\mathbb{C} \ell(4)$ minimal right ideal basis states associated with leptons and quarks. We emphasize however that it is the underlying algebraic structure of the $\mathbb{C} \ell(6)$ and $\mathbb{C} \ell(4)$ minimal ideals that is of primary importance, and not its possible embedding into $\mathbb{C} \ell(10)$.

Although the unitary symmetries that preserves a Witt decomposition of $\mathbb{C} \ell(10)$ generate $S U(5)$, of the $24 S U(5)$ generators only 12 preserve the distinct $\mathbb{C} \ell(6)$ and $\mathbb{C} \ell(4)$ algebraic sectors of our model. It is precisely the remaining 12 generators that correspond to unobserved processes, including proton decay. If one starts with $\mathbb{C} \ell(10)$ as the underlying background structure, such transformations would not be excluded. In our model on the other hand, the full 24 dimensional $S U(5)$ symmetry is never be realized in the first place, meaning that the unphysical transformations are algebraically excluded.

Besides giving a particularly aesthetic embedding of one generations of fermions into minimal ideals of $\mathbb{C} \ell(10)$, our model also provides a clear pathway for extending earlier work [8] which established a curious structural similarity between the basis states of the minimal left ideals of $\mathbb{C} \ell(6)$ and certain braids used in a topological model of leptons and quarks [9]. Incorporating the results of the present paper to extend these earlier results to include the weak interaction will be the focus of another paper [10].

\section{Electrocolor symmetries for one generations of fermions from $\mathbb{C} \ell(6)$}

The algebra $\mathbb{C} \ell(6)$ has just a single irreducible representation, which acts on an eight complex-dimensional space of states. In [2] it was shown that a Witt decomposition of $\mathbb{C} \ell(6)$ decomposes the algebra into minimal left ideals whose basis states transform as a single generation of leptons and quarks under the unbroken electrocolor symmetry $S U(3)_{C} \times U(1)_{E M}$. Such a Witt basis of $\mathbb{C} \ell(6)$ can be defined as $^{2}$

$\alpha_{1} \equiv \frac{1}{2}\left(-e_{5}+i e_{4}\right), \quad \alpha_{1}^{\dagger} \equiv \frac{1}{2}\left(e_{5}+i e_{4}\right)$,

$\alpha_{2} \equiv \frac{1}{2}\left(-e_{3}+i e_{1}\right), \quad \alpha_{2}^{\dagger} \equiv \frac{1}{2}\left(e_{3}+i e_{1}\right)$,

$\alpha_{3} \equiv \frac{1}{2}\left(-e_{6}+i e_{2}\right), \quad \alpha_{3}^{\dagger} \equiv \frac{1}{2}\left(e_{6}+i e_{2}\right)$,

satisfying the anticommutator algebra of fermionic ladder operators

$\left\{\alpha_{i}^{\dagger}, \alpha_{j}^{\dagger}\right\}=\left\{\alpha_{i}, \alpha_{j}\right\}=0, \quad\left\{\alpha_{i}^{\dagger}, \alpha_{j}\right\}=\delta_{i j}$.

The $\alpha_{i}$ and $\alpha_{i}^{\dagger}$ are nilpotents and each set $\left\{\alpha_{i}\right\}$ and $\left\{\alpha_{i}^{\dagger}\right\}$ generates a maximal totally isotropic subspace of dimension eight. One can then construct the minimal left ideal $S_{0}^{u} \equiv \mathbb{C} \ell(6) \omega \omega^{\dagger}$, where $\omega \omega^{\dagger}=\alpha_{1} \alpha_{2} \alpha_{3} \alpha_{3}^{\dagger} \alpha_{2}^{\dagger} \alpha_{1}^{\dagger}$ is a primitive idempotent. Explicitly:

$$
\begin{aligned}
S^{u} \equiv & v \omega \omega^{\dagger}+\bar{d}^{r} \alpha_{1}^{\dagger} \omega \omega^{\dagger}+\bar{d}^{g} \alpha_{2}^{\dagger} \omega \omega^{\dagger} \\
& +\bar{d}^{b} \alpha_{3}^{\dagger} \omega \omega^{\dagger}+u^{r} \alpha_{3}^{\dagger} \alpha_{2}^{\dagger} \omega \omega^{\dagger}+u^{g} \alpha_{1}^{\dagger} \alpha_{3}^{\dagger} \omega \omega^{\dagger} \\
& +u^{b} \alpha_{2}^{\dagger} \alpha_{1}^{\dagger} \omega \omega^{\dagger}+e^{+} \alpha_{3}^{\dagger} \alpha_{2}^{\dagger} \alpha_{1}^{\dagger} \omega \omega^{\dagger},
\end{aligned}
$$

where $v, \bar{d}^{r}$ etc. are suggestively labeled complex coefficients denoting the isospin-up elementary fermions. The conjugate system analogously gives a second linearly independent minimal left ideal of isospin-down elementary fermions $S^{d} \equiv \mathbb{C} \ell(6) \omega^{\dagger} \omega$. Transformations of ladder operators induce transformations of the states. The representations of the minimal ideals are invariant under the electrocolor symmetry $S U(3)_{C} \times U(1)_{E M}$, whose generators are constructed from the bivectors of the algebra. Each basis state in the ideals transforms as a specific lepton or quark as indicated by its suggestively labeled complex coefficients. That is, the unitary transformations that preserve the Witt decomposition are given by $S U(3)_{C} \times U(1)_{E M}$.

\footnotetext{
${ }^{2}$ Following the convention of [2].
} 
In terms of the Witt basis ladder operators, the $S U(3)_{C}$ generators take the form

$\Lambda_{1}=-\alpha_{2}^{\dagger} \alpha_{1}-\alpha_{1}^{\dagger} \alpha_{2}$,

$\Lambda_{2}=i \alpha_{2}^{\dagger} \alpha_{1}-i \alpha_{1}^{\dagger} \alpha_{2}$,

$\Lambda_{3}=\alpha_{2}^{\dagger} \alpha_{2}-\alpha_{1}^{\dagger} \alpha_{1}$,

$\Lambda_{4}=-\alpha_{1}^{\dagger} \alpha_{3}-\alpha_{3}^{\dagger} \alpha_{1}$,

$\Lambda_{5}=-i \alpha_{1}^{\dagger} \alpha_{3}+i \alpha_{3}^{\dagger} \alpha_{1}$,

$\Lambda_{6}=-\alpha_{3}^{\dagger} \alpha_{2}-\alpha_{2}^{\dagger} \alpha_{3}$,

$\Lambda_{7}=i \alpha_{3}^{\dagger} \alpha_{2}-i \alpha_{2}^{\dagger} \alpha_{3}$,

$\Lambda_{8}=\frac{-1}{\sqrt{3}}\left(\alpha_{1}^{\dagger} \alpha_{1}+\alpha_{2}^{\dagger} \alpha_{2}-2 \alpha_{3}^{\dagger} \alpha_{3}\right)$.

The $U(1)_{E M}$ generator, proportional to the number operator, can be expressed in terms of the Witt basis ladder operators as

$Q=\frac{1}{3}\left(\alpha_{1}^{\dagger} \alpha_{1}+\alpha_{2}^{\dagger} \alpha_{2}+\alpha_{3}^{\dagger} \alpha_{3}\right)$,

and gives the electric charge of fermions.

As an illustrative example we consider $\left[\Lambda_{1}, u^{g}\right]$ :

$$
\begin{aligned}
{\left[\Lambda_{1}, u^{g}\right]=} & \left(-\alpha_{2}^{\dagger} \alpha_{1}-\alpha_{1}^{\dagger} \alpha_{2}\right) \alpha_{1}^{\dagger} \alpha_{3}^{\dagger} \omega \omega^{\dagger} \\
& -\alpha_{1}^{\dagger} \alpha_{3}^{\dagger} \omega \omega^{\dagger}\left(-\alpha_{2}^{\dagger} \alpha_{1}-\alpha_{1}^{\dagger} \alpha_{2}\right), \\
= & -\alpha_{2}^{\dagger} \alpha_{1} \alpha_{1}^{\dagger} \alpha_{3}^{\dagger} \omega \omega^{\dagger}, \\
= & \alpha_{3}^{\dagger} \alpha_{2}^{\dagger} \omega \omega^{\dagger}=u^{r} .
\end{aligned}
$$

We see that these $S U$ (3) generators transform between different basis states of the same ideal, and never transform between ideals. In other words, the set of lowering operators and the set of raising operators are each closed under the $S U(3)_{C} \times U(1)_{E M}$ symmetries.

\section{Weak symmetries for one generation of fermions from $\mathbb{C} \ell(4)$}

\subsection{Towards the weak force}

So far we have considered two minimal left ideals of $\mathbb{C} \ell(6)$. One ideal, $S^{u}$, consists of isospin-up states, and the other, $S^{d}$ consists of isospin-down states. The unitary symmetries $S U(3)_{C}$ and $U(1)_{E M}$ facilitate transitions between states within an ideal, meaning the ideals are closed under the action of the gauge symmetries. On the other hand, multiplying the ideal $S^{u}$ on the right by $\omega$ changes the ideal into $S^{d}$. The same is true for multiplying $S^{d}$ on the right by $\omega^{\dagger}$. Notice that this only works via right-multiplications, not via leftmultiplication. Together, $\omega$ and $\omega^{\dagger}$ generate a copy of $\mathbb{C} \ell(2)$, which is a subalgebra of $\mathbb{C} \ell(6)$. The $S U(2)_{\omega}$ generators are written as follows

$\tau_{1} \equiv \omega+\omega^{\dagger}, \quad \tau_{2} \equiv i\left(\omega-\omega^{\dagger}\right), \quad \tau_{3} \equiv \omega \omega^{\dagger}-\omega^{\dagger} \omega$,

where it needs to be remembered that this $S U(2)_{\omega}$ acts from the right so that $\left[\frac{\tau_{j}}{2}, \frac{\tau_{i}}{2}\right]=i \epsilon_{i j k} \frac{\tau_{k}}{2}$. This $S U(2)_{\omega}$ symmetry transforms between states of different ideals. In particular, note that the action of this $S U(2)_{\omega}$ onto a state changes its electric charge by plus or minus one. This $S U(2)_{\omega}$ does not preserve the $\mathbb{C} \ell(6)$ minimal left ideals and so is not interpreted as a gauge symmetry. Nonetheless, this $\mathbb{C} \ell(2)$ plays an important role in weak interactions since a weak doublet contains two particles that belong to different $\mathbb{C} \ell(6)$ minimal left ideals and differ in their electric charge by one. To describe the $S U(2)_{L}$ weak symmetry, we want to include an additional Clifford algebra, whose Witt decomposition is preserved by $S U(2)$. This algebra is $\mathbb{C} \ell(4)$.

3.2 Minimal right ideals of $\mathbb{C} \ell(4)$ and unitary symmetries

Similarly to $\mathbb{C} \ell(6), \mathbb{C} \ell(4)$ has just a single irreducible representation, which acts on a four complex-dimensional space of states. $\mathbb{C} \ell(4)$, with say orthonormal basis $\left\{w_{1}, w_{2}, w_{3}, w_{4}\right\}$, may be rewritten in terms of a nilpotent basis $\left\{\beta_{1}, \beta_{2}, \beta_{1}^{\dagger}, \beta_{2}^{\dagger}\right\}$ of ladder operators. Following the construction of minimal left ideals of $\mathbb{C} \ell(6)$, we construct the minimal right ideals of $\mathbb{C} \ell(4)$. First define the nilpotents $\Omega=\beta_{2} \beta_{1}$ and $\Omega^{\dagger}=\beta_{1}^{\dagger} \beta_{2}^{\dagger}$, from which one constructs the idempotents $\Omega \Omega^{\dagger}$ and $\Omega^{\dagger} \Omega$. Two minimal right ideals are then given by $\Omega \Omega^{\dagger} \mathbb{C} \ell(4)$ and $\Omega^{\dagger} \Omega \mathbb{C} \ell(4)$, which are four complex-dimensions. Explicitly the ideals are spanned by the states

$$
\begin{aligned}
& \left\{\Omega \Omega^{\dagger}, \Omega \Omega^{\dagger} \beta_{1}, \Omega \Omega^{\dagger} \beta_{2}, \Omega \Omega^{\dagger} \beta_{2} \beta_{1}\right\}, \\
& \left\{\Omega^{\dagger} \Omega, \Omega^{\dagger} \Omega \beta_{1}^{\dagger}, \Omega^{\dagger} \Omega \beta_{2}^{\dagger}, \Omega^{\dagger} \Omega \beta_{1}^{\dagger} \beta_{2}^{\dagger}\right\} .
\end{aligned}
$$

The $S U(2)$ and $U(1)$ unitary symmetries that preserve the ladder operator basis of $\mathbb{C} \ell(4)$ are generated by [11]:

$$
\begin{aligned}
& T_{1}=-\beta_{1} \beta_{2}^{\dagger}-\beta_{2} \beta_{1}^{\dagger}, \\
& T_{2}=i \beta_{1} \beta_{2}^{\dagger}-i \beta_{2} \beta_{1}^{\dagger}, \\
& T_{3}=\beta_{1} \beta_{1}^{\dagger}-\beta_{2} \beta_{2}^{\dagger}, \\
& N=\beta_{1} \beta_{1}^{\dagger}+\beta_{2} \beta_{2}^{\dagger} .
\end{aligned}
$$

Under the action of the $S U$ (2) generators the states $\Omega^{\dagger} \Omega$ and $\Omega^{\dagger} \Omega \beta_{1}^{\dagger} \beta_{2}^{\dagger}$ transform as singlets whereas the states $\Omega^{\dagger} \Omega \beta_{1}^{\dagger}$ and $\Omega^{\dagger} \Omega \beta_{2}^{\dagger}$ transform into each other as a doublet. The singlet states may then be identified with right handed fermions, and the doublet states with left-handed fermions. Similarly, the states $\Omega \Omega^{\dagger}$ and $\Omega \Omega^{\dagger} \beta_{1} \beta_{2}$ are identified with left-handed anti-fermions, whereas $\Omega \Omega^{\dagger} \beta_{1}$ and $\Omega \Omega^{\dagger} \beta_{2}$ with right-handed anti-fermions. It is again an important point that the $\mathbb{C} \ell(4)$ ideals are closed under the action of 
this $S U(2) \times U(1)$. The generators (9) transform between different basis states of the same $\mathbb{C} \ell(4)$ ideal.

Subsequently, we can write two $\mathbb{C} \ell(4)$ minimal right ideals, one for leptons and one for anti-leptons, as

$L=v_{R} \Omega^{\dagger} \Omega+v_{L} \Omega^{\dagger} \Omega \beta_{1}^{\dagger}+e_{L}^{-} \Omega^{\dagger} \Omega \beta_{2}^{\dagger}+e_{R}^{-} \Omega^{\dagger} \Omega \beta_{1}^{\dagger} \beta_{2}^{\dagger}$,

and

$\bar{L}=\bar{v}_{L} \Omega \Omega^{\dagger}+\bar{v}_{R} \Omega \Omega^{\dagger} \beta_{1}+e_{R}^{+} \Omega \Omega^{\dagger} \beta_{2}+e_{L}^{+} \Omega \Omega^{\dagger} \beta_{2} \beta_{1}$,

where in both cases the coefficients are complex numbers indicating the particle it transforms like.

As an example, consider $\left[T_{1}, v_{L}\right]$ :

$$
\begin{aligned}
{\left[T_{1}, v_{L}\right]=} & \left(-\beta_{1} \beta_{2}^{\dagger}-\beta_{2} \beta_{1}^{\dagger}\right) \Omega^{\dagger} \Omega \beta_{1}^{\dagger} \\
& -\Omega^{\dagger} \Omega \beta_{1}^{\dagger}\left(-\beta_{1} \beta_{2}^{\dagger}-\beta_{2} \beta_{1}^{\dagger}\right), \\
= & -\Omega^{\dagger} \Omega \beta_{1}^{\dagger}\left(-\beta_{1} \beta_{2}^{\dagger}\right), \\
= & \Omega^{\dagger} \Omega \beta_{2}^{\dagger}=e_{L} .
\end{aligned}
$$

Similarly, for the quarks and anti-quarks, the $\mathbb{C} \ell(4) \min -$ imal right ideals can be written as

$$
\begin{aligned}
Q^{(3)}= & u_{R}^{(3)} \Omega^{\dagger} \Omega+u_{L}^{(3)} \Omega^{\dagger} \Omega \beta_{1}^{\dagger} \\
& +d_{L}^{(3)} \Omega^{\dagger} \Omega \beta_{2}^{\dagger}+d_{R}^{(3)} \Omega^{\dagger} \Omega \beta_{1}^{\dagger} \beta_{2}^{\dagger},
\end{aligned}
$$

and

$$
\begin{aligned}
\bar{Q}^{(3)}= & \bar{u}_{L}^{(3)} \Omega \Omega^{\dagger}+\bar{u}_{R}^{(3)} \Omega \Omega^{\dagger} \beta_{1} \\
& +\bar{d}_{R}^{(3)} \Omega \Omega^{\dagger} \beta_{2}+\bar{d}_{L}^{(3)} \Omega \Omega^{\dagger} \beta_{2} \beta_{1},
\end{aligned}
$$

where the superscripts refers to the color of the quarks. Presently, as $\mathbb{C} \ell(4)$ minimal right ideals, the quark ideals and lepton ideals are identical. We will be able to distinguish between leptons and quarks once we include the $\mathbb{C} \ell(6)$ minimal left ideals.

\section{Combining $\mathbb{C} \ell(6)$ electrocolor and $\mathbb{C} \ell(4)$ weak states}

We now combine the earlier results based on $\mathbb{C} \ell(6)$ electrocolor states with the $\mathbb{C} \ell(4)$ weak states of the preceding section. We assume for simplicity here that the two algebras commute so that $\alpha_{i} \beta_{j}=\beta_{j} \alpha_{i}$. Everything that follows still works, with some minor modifications, when $\alpha_{i}$ and $\beta_{j}$ anticommute.

The neutrino $v$ is represented by the $\mathbb{C} \ell(6)$ minimal left ideal basis state $\omega \omega^{\dagger}$. Via the $\mathbb{C} \ell(4)$ right ideals of the previous section, we can now include chirality. We then have

$v_{R}=\omega \omega^{\dagger} \Omega^{\dagger} \Omega$,

$v_{L}=\omega \omega^{\dagger} \Omega^{\dagger} \Omega \beta_{1}^{\dagger}$.
Similarly, the neutrino's weak doublet partner, the electron $e^{-}$in its left- and right-handed form can now be written as

$e_{L}^{-}=\alpha_{1} \alpha_{2} \alpha_{3} \omega^{\dagger} \omega \Omega^{\dagger} \Omega \beta_{2}^{\dagger}$,

$e_{R}^{-}=\alpha_{1} \alpha_{2} \alpha_{3} \omega^{\dagger} \omega \Omega^{\dagger} \Omega \beta_{1}^{\dagger} \beta_{2}^{\dagger}$.

It is important to notice that the neutrino and electron live in different $\mathbb{C} \ell(6)$ minimal left ideals, but in the same $\mathbb{C} \ell(4)$ minimal right ideal.

The red up quark $u^{r}$ with electrocolor symmetry was previously identified with the $\mathbb{C} \ell(6)$ state $\alpha_{3}^{\dagger} \alpha_{2}^{\dagger} \omega \omega^{\dagger}$. Via the $\mathbb{C} \ell(4)$ right ideals of the previous section, we can now include chirality. We then have

$u_{R}^{r}=\alpha_{3}^{\dagger} \alpha_{2}^{\dagger} \omega \omega^{\dagger} \Omega^{\dagger} \Omega$,

$u_{L}^{r}=\alpha_{3}^{\dagger} \alpha_{2}^{\dagger} \omega \omega^{\dagger} \Omega^{\dagger} \Omega \beta_{1}^{\dagger}$,

Subsequently, the red down quark $d^{r}$ becomes

$d_{L}^{r}=\alpha_{1} \omega^{\dagger} \omega \Omega^{\dagger} \Omega \beta_{2}^{\dagger}$,

$d_{R}^{r}=\alpha_{1} \omega^{\dagger} \omega \Omega^{\dagger} \Omega \beta_{1}^{\dagger} \beta_{2}^{\dagger}$.

Again, the red down quark and red up quark belong to different $\mathbb{C} \ell(6)$ ideals but the same $\mathbb{C} \ell(4)$ ideal. The antiparticles live in the conjugate $\mathbb{C} \ell(4)$ minimal right ideal. Hence, for example

$e_{R}^{+}=\alpha_{3}^{\dagger} \alpha_{2}^{\dagger} \alpha_{1}^{\dagger} \omega \omega^{\dagger} \Omega \Omega^{\dagger} \beta_{2}$,

$\bar{u}_{L}^{r}=\alpha_{2} \alpha_{3} \omega^{\dagger} \omega \Omega \Omega^{\dagger}$.

In summary, the eight weak-doublets are identified as

$$
\begin{aligned}
\left(\begin{array}{c}
v_{L} \\
e_{L}^{-}
\end{array}\right) & =\left(\begin{array}{c}
\omega \omega^{\dagger} \Omega^{\dagger} \Omega \beta_{1}^{\dagger} \\
\alpha_{1} \alpha_{2} \alpha_{3} \omega^{\dagger} \omega \Omega^{\dagger} \Omega \beta_{2}^{\dagger}
\end{array}\right), \\
\left(\begin{array}{c}
u_{L}^{(3)} \\
d_{L}^{(3)}
\end{array}\right) & =\left(\begin{array}{c}
\alpha_{j}^{\dagger} \alpha_{i}^{\dagger} \omega \omega^{\dagger} \Omega^{\dagger} \Omega \beta_{1}^{\dagger} \\
\epsilon_{i j k} \alpha_{k} \omega^{\dagger} \omega \Omega^{\dagger} \Omega \beta_{2}^{\dagger}
\end{array}\right), \\
\left(\begin{array}{c}
e_{R}^{+} \\
\bar{v}_{R}
\end{array}\right) & =\left(\begin{array}{c}
\alpha_{3}^{\dagger} \alpha_{2}^{\dagger} \alpha_{1}^{\dagger} \omega \omega^{\dagger} \Omega \Omega^{\dagger} \beta_{2} \\
\omega^{\dagger} \omega \Omega \Omega^{\dagger} \beta_{1}
\end{array}\right), \\
\left(\begin{array}{c}
\bar{d}_{R}^{(3)} \\
\bar{u}_{R}^{(3)}
\end{array}\right) & =\left(\begin{array}{c}
\alpha_{i}^{\dagger} \omega \omega^{\dagger} \Omega \Omega^{\dagger} \beta_{2} \\
\epsilon_{i j k} \alpha_{j} \alpha_{k} \omega^{\dagger} \omega \Omega \Omega^{\dagger} \beta_{1}
\end{array}\right)
\end{aligned}
$$

All of the other physical states are weak singlets

$$
\begin{aligned}
\left(v_{R}\right) & =\left(\omega \omega^{\dagger} \Omega^{\dagger} \Omega\right),\left(e_{R}^{-}\right)=\left(\alpha_{1} \alpha_{2} \alpha_{3} \omega^{\dagger} \omega \Omega^{\dagger} \Omega \beta_{1}^{\dagger} \beta_{2}^{\dagger}\right), \\
\left(e_{L}^{+}\right) & =\left(\alpha_{3}^{\dagger} \alpha_{2}^{\dagger} \alpha_{1}^{\dagger} \omega \omega^{\dagger} \Omega \Omega^{\dagger} \beta_{2} \beta_{1}\right),\left(\bar{v}_{L}\right)=\left(\omega^{\dagger} \omega \Omega \Omega^{\dagger}\right), \\
\left(u_{R}^{(3)}\right) & =\left(\alpha_{j}^{\dagger} \alpha_{i}^{\dagger} \omega \omega^{\dagger} \Omega^{\dagger} \Omega\right), \\
\left(d_{R}^{(3)}\right) & =\left(\epsilon_{i j k} \alpha_{k} \omega^{\dagger} \omega \Omega^{\dagger} \Omega \beta_{1}^{\dagger} \beta_{2}^{\dagger}\right), \\
\left(\bar{d}_{L}^{(3)}\right) & =\left(\alpha_{i}^{\dagger} \omega \omega^{\dagger} \Omega \Omega^{\dagger} \beta_{2} \beta_{1}\right), \\
\left(\bar{u}_{L}^{(3)}\right) & =\left(\epsilon_{i j k} \alpha_{j} \alpha_{k} \omega^{\dagger} \omega \Omega \Omega^{\dagger}\right) .
\end{aligned}
$$




\section{Weak $S U(2)_{L}$ symmetries}

Now that we can write down chiral fermions in terms of $\mathbb{C} \ell(6)$ and $\mathbb{C} \ell(4)$ minimal ideals, we must next find appropriate $S U$ (2) generators so that the states transform correctly via the weak symmetry $S U(2)_{L}$.

Consider the weak doublet consisting of a left handed neutrino and left handed electron. In terms of the ideals we have:

$$
\left(\begin{array}{c}
v_{L} \\
e_{L}^{-}
\end{array}\right)=\left(\begin{array}{c}
\omega \omega^{\dagger} \Omega^{\dagger} \Omega \beta_{1}^{\dagger} \\
\alpha_{1} \alpha_{2} \alpha_{3} \omega^{\dagger} \omega \Omega^{\dagger} \Omega \beta_{2}^{\dagger}
\end{array}\right)
$$

To transform the neutrino into the electron requires not only that $\beta_{1}^{\dagger}$ is transformed into $\beta_{2}^{\dagger}$ via $T_{i}$ in Eq. (9), but also that the $\mathbb{C} \ell(6)$ ideal, and electric charge are changed. The latter two transformations are mediated by the $S U(2)_{\omega}$ generators (6). What is required in the present case then is a combination of the generators (9) and (6). ${ }^{3}$ After some deliberation, the suitable $S U(2)_{L}$ generators can be chosen as

$$
\begin{aligned}
& T_{1}^{\prime} \equiv-\beta_{1} \beta_{2}^{\dagger} \omega-\beta_{2} \beta_{1}^{\dagger} \omega^{\dagger}, \\
& T_{2}^{\prime} \equiv i \beta_{1} \beta_{2}^{\dagger} \omega-i \beta_{2} \beta_{1}^{\dagger} \omega^{\dagger}, \\
& T_{3}^{\prime} \equiv \beta_{1} \beta_{2}^{\dagger} \beta_{2} \beta_{1}^{\dagger} \omega \omega^{\dagger}-\beta_{2} \beta_{1}^{\dagger} \beta_{1} \beta_{2}^{\dagger} \omega^{\dagger} \omega .
\end{aligned}
$$

As a first example, consider the action of $T_{1}^{\prime}$ on the lefthanded electron $v_{L}$

$$
\begin{aligned}
{\left[T_{1}^{\prime}, v_{L}\right]=} & -\left(\beta_{1} \beta_{2}^{\dagger} \omega+\beta_{2} \beta_{1}^{\dagger} \omega^{\dagger}\right)\left(\omega \omega^{\dagger} \Omega^{\dagger} \Omega \beta_{1}^{\dagger}\right) \\
& +\left(\omega \omega^{\dagger} \Omega^{\dagger} \Omega \beta_{1}^{\dagger}\right)\left(\beta_{1} \beta_{2}^{\dagger} \omega+\beta_{2} \beta_{1}^{\dagger} \omega^{\dagger}\right), \\
= & 0+\omega \omega^{\dagger} \Omega^{\dagger} \Omega \beta_{1}^{\dagger} \beta_{1} \beta_{2}^{\dagger} \omega, \\
= & \omega \omega^{\dagger} \omega \Omega^{\dagger} \Omega \beta_{2}^{\dagger}, \\
= & \alpha_{1} \alpha_{2} \alpha_{3} \omega^{\dagger} \omega \Omega^{\dagger} \Omega \beta_{2}^{\dagger}=e_{L}^{-} .
\end{aligned}
$$

Furthermore, $\left[T_{3}^{\prime}, v_{L}\right]=-v_{L}$, so that $\hat{T}_{3}=-\frac{1}{2} T_{3}^{\prime}$ returns the correct weak isospin of physical states.

As a second example consider an anti-quark weak doublet.

$$
\left(\begin{array}{c}
\bar{d}_{R}^{r} \\
\bar{u}_{R}^{r}
\end{array}\right)=\left(\begin{array}{c}
\alpha_{1}^{\dagger} \omega \omega^{\dagger} \Omega \Omega^{\dagger} \beta_{2} \\
\alpha_{2} \alpha_{3} \omega^{\dagger} \omega \Omega \Omega^{\dagger} \beta_{1}
\end{array}\right)
$$

Then,

$$
\begin{aligned}
{\left[T_{1}^{\prime}, \bar{u}_{R}^{r}\right] } & =-\left(\alpha_{2} \alpha_{3} \omega^{\dagger} \omega \Omega \Omega^{\dagger} \beta_{1}\right)\left(-\beta_{1} \beta_{2}^{\dagger} \omega-\beta_{2} \beta_{1}^{\dagger} \omega^{\dagger}\right), \\
& =\left(-\alpha_{2} \alpha_{3} \omega^{\dagger}\right) \omega \omega^{\dagger} \Omega \Omega^{\dagger} \beta_{2}, \\
& =-\alpha_{1}^{\dagger} \omega \omega^{\dagger} \Omega \Omega^{\dagger} \beta_{2}=-\bar{d}_{R}^{r} .
\end{aligned}
$$

The key point is that the generators (29) simultaneously transform both within a $\mathbb{C} \ell(4)$ ideal and between $\mathbb{C} \ell(6)$ ideals,

\footnotetext{
${ }^{3}$ Herein lies the unique approach of this paper. In [7], the weak interaction are generated simply by (9). This leaves the $\mathbb{C} \ell(6)$ unaffected and the result is that the electron is then represented as $\omega \omega^{\dagger} \Omega^{\dagger} \Omega \beta_{2}$.
}

and the set of physical states is closed under the action of this symmetry.

\section{$6 \mathbb{C} \ell(10)$ and $S U(5)$ GUT}

Combining the $\mathbb{C} \ell(6)$ minimal left ideals and $\mathbb{C} \ell(4)$ minimal right ideals, it is possible to rewrite physical states as basis states of a single $\mathbb{C} \ell(10)$ minimal left ideal, as is done in [7]. Given our different $S U(2)_{L}$ generators, the physical states in our model will be represented differently, and most naturally belong to two different $\mathbb{C} \ell(10)$ ideals.

Consider the left handed electron. In [7] this state is represented as $e_{L}^{-}=\beta_{2}^{\dagger} \omega^{\dagger} \omega \Omega \Omega^{\dagger}$, where the notation has been adopted to be consistent with the present paper. One sees, due to the lack of $\alpha_{1} \alpha_{2} \alpha_{3}$ that the original $\mathbb{C} \ell(6)$ representation of the electron is not preserved in this construction. Consequently, $Q$ no longer gives the electric charge in this $\mathbb{C} \ell(10)$ scheme. On the other hand, our scheme identifies the lefthanded electron as $e_{L}^{-}=\alpha_{1} \alpha_{2} \alpha_{3} \omega^{\dagger} \omega \Omega^{\dagger} \Omega \beta_{2}^{\dagger}$, which may be rewritten as a $\mathbb{C} \ell(10)$ element as $e_{L}^{-}=\alpha_{1} \alpha_{2} \alpha_{3} \beta_{2}^{\dagger} \omega^{\dagger} \omega \Omega \Omega^{\dagger}$. This representation preserves both the electrocolor structure $\alpha_{1} \alpha_{2} \alpha_{3}$ from $\mathbb{C} \ell(6)$ and the weak structure $\beta_{2}^{\dagger}$ from $\mathbb{C} \ell(4)$. Subsequently $Q$ still gives the correct electric charge of the electron.

The unitary symmetry that preserves a Witt decomposition of $\mathbb{C} \ell(10)$ algebra is $S U(5)$, the basis of the Georgi and Glashow GUT. This GUT predicts additional gauge bosons and associated unobserved physical processes, most famously proton decay. However, in the present case, physical states belong simultaneously to a minimal left ideal of $\mathbb{C} \ell(6)$ and a minimal right ideal of $\mathbb{C} \ell(4)$. Although our physical states can be rewritten as basis elements of minimal left ideals of $\mathbb{C} \ell(10)$ they do not correspond to the minimal ideals obtained from a direct Witt decomposition of $\mathbb{C} \ell(10){ }^{4}$ Importantly, unitary symmetries of the form $\alpha_{i} \beta_{j}^{\dagger}+\beta_{j} \alpha^{\dagger}$ which preserve the minimal left ideals obtained from a Witt decomposition of $\mathbb{C} \ell(10)$ are naturally excluded in our model because they inevitably mix the distinct $\mathbb{C} \ell(6)$ and $\mathbb{C} \ell(4)$ algebraic sectors. It is precisely these $S U(5)$ symmetries that enable unobserved physical processes, and these are therefore algebraically excluded in our construction. In our discussion, the full $S U(5)$ symmetry of the $\mathbb{C} \ell(10)$ minimal ideals should never be fully realized because it is the $\mathbb{C} \ell(6)$ and $\mathbb{C} \ell(4)$ minimal ideals that are of primary importance, not their embedding into $\mathbb{C} \ell(10)$.

\footnotetext{
${ }^{4}$ This is easy to see. The $\alpha_{1} \alpha_{2} \alpha_{3} \beta_{2}^{\dagger}$ factor in the state $e_{L}^{-}=$ $\alpha_{1} \alpha_{2} \alpha_{3} \beta_{2}^{\dagger} \omega^{\dagger} \omega \Omega \Omega^{\dagger}$ combines both the lowering operators from $\mathbb{C} \ell(6)$ and the raising operators of $\mathbb{C} \ell(4)$. The standard construction of ideals from a Witt decomposition gives one ideal constructed entirely from raising operators acting on a primitive idempotent, and a second ideal constructed entirely from lowering operators acting on a primitive idempotent.
} 


\section{Discussion}

Two minimal left ideals of $\mathbb{C} \ell(6)$, and two minimal right ideals of $\mathbb{C} \ell(4)$ were shown to transform as a generation of fermions under the unbroken electrocolor group $S U(3)_{C} \times$ $U(1)_{E M}$, and the weak group $S U(2)_{L}$ respectively. Combining the $\mathbb{C} \ell(6)$ and $\mathbb{C} \ell(4)$ ideal basis states, the leptons and quarks for a single generation transforming correctly under the SM gauge group can be represented. These combined $\mathbb{C} \ell(6)$ and $\mathbb{C} \ell(4)$ ideal basis states can be embedded into minimal left ideals of $\mathbb{C} \ell(10)$ in such a way that preserves individually the $\mathbb{C} \ell(6)$ structure and $\mathbb{C} \ell(4)$ structure of physical states. This is an improvement over an earlier model [7] where the individual $\mathbb{C} \ell(6)$ structure and $\mathbb{C} \ell(4)$ structure of physical states is not preserved. This improvement is achieved through redefining the $S U(2)_{L}$ to include factors of $\omega$ and $\omega^{\dagger}$ in appropriate places to ensure that a transformation within a $\mathbb{C} \ell(4)$ minimal right ideal simultaneously maps between different $\mathbb{C} \ell(6)$ minimal left ideals.

It is important to notice the conceptual difference between the $S U$ (2) generated from (6) and the $S U(2)$ generated from (9). In the former case, $\omega$ and $\omega^{\dagger}$ span a $\mathbb{C} \ell(2)$ subalgebra of $\mathbb{C} \ell(6)$. This algebra is isomorphic to $S U(2)$ and the generators mediate transitions between the $\mathbb{C} \ell(6)$ ideals $S^{u}$ and $S^{d}$. This $S U(2)$ symmetry is not a gauge symmetry as it does not transform between states within the same ideal, but rather between states of different ideals. In the latter case, the $S U(2)$ generators are constructed from the bivectors of $\mathbb{C} \ell(4)$. This copy of $S U$ (2) contains the symmetries that preserves the Witt decomposition of $\mathbb{C} \ell(4)$, and so correspond to a gauge symmetry.

In our model, unitary symmetries generated from combinations of $\mathbb{C} \ell(6)$ and $\mathbb{C} \ell(4)$ ladder operators, such as $\alpha_{i} \beta_{j}^{\dagger}+\beta_{j} \alpha^{\dagger}$, are naturally excluded because they do not preserve the individual $\mathbb{C} \ell(6)$ and $\mathbb{C} \ell(4)$ structure. It is precisely these types of generators that are responsible for the unobserved particle processes, including proton decay, in the Georgi-Glashow $S U$ (5) GUT. In our model, the $S U(5)$ gauge symmetry is thus never fully realized in the first place.

The motivation for this work came from the results of [8] which showed that there is a one-to-one correspondence between the basis states of the minimal left ideals of $\mathbb{C} \ell(6)$ and the braided states in the topological model proposed in [9]. ${ }^{5}$ To be able to extend this curious structural similarity to include the weak force, the underlying electrocolor structure generated from $\mathbb{C} \ell(6)$ should remain intact. This is not the case in the $\mathbb{C} \ell(10)$ representation in [7], but is the case for the construction considered here. The extension of [8] to include the weak force will be the focus of an upcoming paper [10].

\footnotetext{
${ }^{5}$ For additional work related to this braid model and its connections with division algebras and Clifford algebras, the reader is referred to [12-18].
}

Acknowledgements This work is supported by the Natural Science Foundation of the Jiangsu Higher Education Institutions of China Programme Grant 19KJB140018 and XJTLU REF-18-02-03 Grant.

Data Availability Statement This manuscript has no associated data or the data will not be deposited. [Authors' comment: This is a theoretical study and no experimental data has been listed.]

Open Access This article is licensed under a Creative Commons Attribution 4.0 International License, which permits use, sharing, adaptation, distribution and reproduction in any medium or format, as long as you give appropriate credit to the original author(s) and the source, provide a link to the Creative Commons licence, and indicate if changes were made. The images or other third party material in this article are included in the article's Creative Commons licence, unless indicated otherwise in a credit line to the material. If material is not included in the article's Creative Commons licence and your intended use is not permitted by statutory regulation or exceeds the permitted use, you will need to obtain permission directly from the copyright holder. To view a copy of this licence, visit http://creativecomm ons.org/licenses/by/4.0/.

Funded by $\mathrm{SCOAP}^{3}$.

\section{References}

1. H. Georgi, S.L. Glashow, Unity of all elementary-particle forces. Phys. Rev. Lett. 32(8), 438 (1974)

2. C. Furey, Standard model physics from an algebra? (2016). arXiv: 1611.09182

3. M. Günaydin, F. Gürsey, Quark structure and octonions. J. Math. Phys. 14(11), 1651-1667 (1973)

4. A.B. Gillard, N.G. Gresnigt, Three fermion generations with two unbroken gauge symmetries from the complex sedenions. Eur. Phys. J. C 79(5), 446 (2019)

5. G.M. Dixon, Division Algebras: Octonions Quaternions Complex Numbers and the Algebraic Design of Physics, vol. 290 (Springer Science \& Business Media, Berlin, 2013)

6. C.C. Perelman, $\mathbb{R} \otimes \mathbb{C} \otimes \mathbb{H} \otimes \mathbb{O}$-valued gravity as a grand unified field theory. Adv. Appl. Clifford Algebras 29(1), 22 (2019)

7. C. Furey, $S U(3)_{C} \times S U(2)_{L} \times U(1)_{Y}\left(\times U(1)_{X}\right)$ as a symmetry of division algebraic ladder operators. Eur. Phys. J. C 78(5), 375 (2018)

8. N.G. Gresnigt, Braids, normed division algebras, and standard model symmetries. Phys. Lett. B 783, 212-221 (2018)

9. S.O. Bilson-Thompson, A topological model of composite preons (2005). arXiv:hep-ph/0503213

10. N.G. Gresnigt, A topological model of composite preons from the minimal ideals of two Clifford algebras (2020). arXiv:2004.11140

11. C. Furey, A demonstration that electroweak theory can violate parity automatically (leptonic case). Int. J. Mod. Phys. A 33(04), 1830005 (2018)

12. S.O. Bilson-Thompson, F. Markopoulou, L. Smolin, Quantum gravity and the standard model. Class. Quantum Gravity 24(16), 3975 (2007)

13. S. Bilson-Thompson, J. Hackett, L.H. Kauffman, Particle topology, braids, and braided belts. J. Math. Phys. 50(11), 113505 (2009)

14. N.G. Gresnigt, Braided fermions from Hurwitz algebras. J. Phys. Conf. Ser. 1194, 012040 (2019)

15. N.G. Gresnigt, Quantum groups and braid groups as fundamental symmetries (2017). arXiv:1711.09011v1

16. N. Gresnigt, A combing algorithm for orientable braided 3-belts. J. Math. Phys. 60(11), 113502 (2019) 
17. T. Asselmeyer-Maluga, Braids, 3-manifolds, elementary particles: number theory and symmetry in particle physics. Symmetry 11(10), 1298 (2019)
18. D. Cartin, Braids as a representation space of su (5). J. Math. Phys. 56(6), 061703 (2015) 